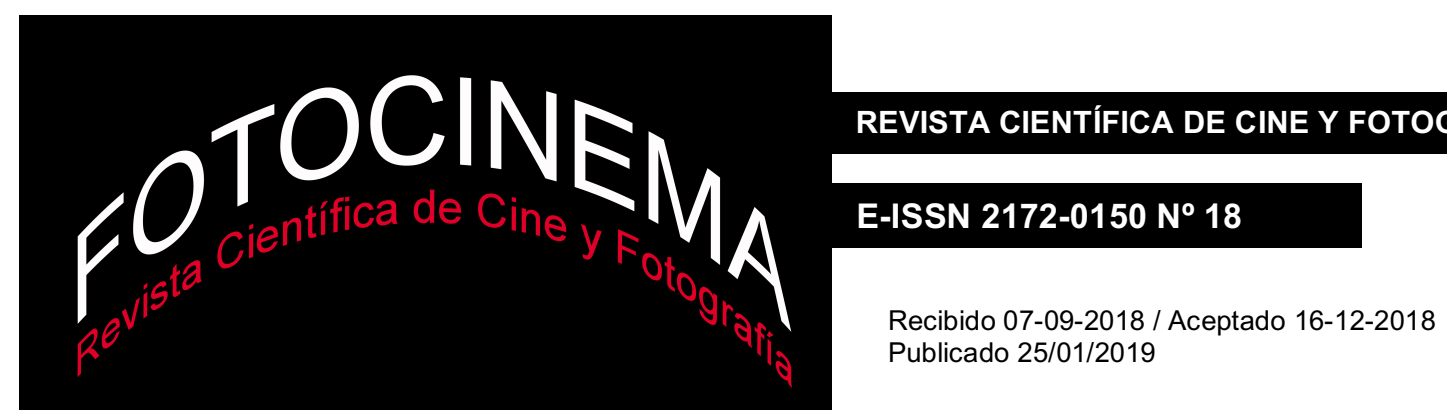

\title{
El bloqueo del escritor en Días sin huella (The Lost Weekend, Billy Wilder, 1945)
}

\section{Writer's block in The Lost Weekend (Billy Wilder, 1945)}

\author{
Juan Carlos Pueo \\ Universidad de Zaragoza, España \\ jcpueo@unizar.es
}

\section{Resumen:}

La adaptación de la novela de Charles R. Jackson The Lost Weekend planteó un primer problema a los guionistas Billy Wilder y Charles Brackett: no podían explicar el alcoholismo del protagonista a partir de su homosexualidad. Decidieron, por tanto, desarrollar otro tema, el del bloqueo del escritor. La responsabilidad del escritor sobre su palabra se plantea en la película mediante el relato de su "fin de semana perdido", en el que su visión del mundo se irá matizando a partir de diferentes tipos de escritura, que van de la formada por simples indicios a la más compleja escritura simbólica. El final de la película no plantea tan sólo la redención del alcohólico, sino el aprendizaje del sentido último de la escritura, siempre según la mentalidad dominante en el Hollywood clásico: la creencia de que la escritura sólo tiene sentido cuando su mensaje va más allá de la mera literatura, instando a los lectores / espectadores a reaccionar en un sentido determinado.

\begin{abstract}
:
The adaptation of Charles R. Jackson's novel The Lost Weekend posed a first problem to the writers Billy Wilder and Charles Brackett: they could not explain the alcoholism of the protagonist from his homosexuality. They decided, therefore, to develop another theme, that of the writer's block. The responsibility of the writer on his words is raised in the film through the story of his "lost weekend", in which his vision of the world will be nuanced from different types of writing, ranging from that one formed by simple indexes to the most complex symbolic writing. The end of the film does not only point to the redemption of the alcoholic, but to the learning of the ultimate meaning of writing, always according to the dominant mentality in classical Hollywood: the belief that writing only makes sense when its message goes beyond mere literature, urging readers / viewers to react in a certain way.
\end{abstract}

Palabras clave:

Escritura; bloqueo del escritor; Billy Wilder; Días sin huella.

Keywords:

Writing; writer's block; Billy Wilder; The Lost Weekend. 


\section{o. Prolijo prólogo o proemio: el marco teórico y la metodología}

Para la realización de este estudio se adoptó un marco teórico de carácter eminentemente neoformalista, según los principios expuestos por D. Bordwell $(1985,1989)$ y atento sobre todo a la descripción de los principales rasgos de lo que se denomina el "modo de representación institucional" del cine clásico de Hollywood tal como se describe en Bordwell, Staiger \& Thompson (1985). Partiendo de esta base, se han tenido en cuenta también las aportaciones al estudio del cine hechas desde la semiótica y el psicoanálisis, atendiendo sobre todo a los fundamentales textos de C. Metz (1968-73, 1977), pero también a posibles convergencias con otras formas de interpretación (Žižek, 1991). No se han encontrado antecedentes específicos sobre la relación entre alcoholismo y literatura en la película de Billy Wilder, más allá de la bibliografía crítica sobre el director, donde se mencionan tan sólo de soslayo.

La diversidad de enfoques adoptados ha hecho desaconsejable ceñirse a una metodología específica, si bien el artículo se ha realizado siguiendo estos tres pasos: 1) visionado de la película Días sin huella (The Lost Weekend, Billy Wilder, 1945), atendiendo a la temática objeto de estudio; 2) reflexión sobre los principales aspectos relacionados con la enunciación cinematográfica de los tópicos del alcoholismo y la escritura; 3) puesta por escrito de las conclusiones alcanzadas tras la reflexión en torno a los puntos de la argumentación sustanciales, atendiendo a la bibliografía específica que pudiera aclarar los aspectos determinantes de dicha argumentación.

\section{Introducción: escritura y alcoholismo, una relación peligrosa}

Cuando Billy Wilder y Charles Brackett se enfrentaron a la tarea de adaptar la novela de Charles R. Jackson The Lost Weekend (1944), se encontraron con un grave problema que deberían solventar desde el primer momento: el relato de Jackson, basado en su propia biografía, daba numerosas pistas sobre la causa del alcoholismo del protagonista, una homosexualidad latente que el código Hays no permitiría mostrar, ni siquiera insinuar, en pantalla. El problema se soslayó dando mayor protagonismo a la novia del personaje, de forma que su 
heterosexualidad no pudiese quedar nunca en entredicho. No obstante, era preciso encontrar un motivo plausible que justificase el estrés, la baja autoestima y la patología depresiva que le conducirían al consumo de alcohol ya en fase de adicción incontrolable. Los guionistas encontraron la solución apelando a la condición de escritor de Don Birnam (Ray Milland), bien que escritor fracasado, incapaz de vencer su miedo a la página en blanco, tal como se reconoce ante su enamorada Helen (Jane Wyman):

- HeLEN (H): Pero tienes que beber por alguna razón. Un médico adecuado podría averiguarlo.

-DON (D); Mira, lo mío está fuera del alcance de los médicos. Sé la razón. La razón soy yo: lo que soy. O, mejor dicho, lo que no soy, lo que hubiera querido ser y no fui.

$-\mathrm{H}:$ ¿Qué es lo que tanto deseas ser y no eres?

-D: Escritor. Qué tontería, ¿no? En la universidad me tenían por un genio. No podían sacar la revista de la facultad sin uno de mis artículos. Sí, estaba inspirado. Parecía Hemingway. Alcancé la cima a los diecinueve años: vendí un relato a la Atlantic Monthly y también al Reader's Digest. ¿Quién va a querer seguir en la universidad cuando es un Hemingway? Mi madre me compró una máquina de escribir y me trasladé directamente a Nueva York. El primer relato que escribí no gustó demasiado, y el segundo lo abandoné: el público no estaba preparado para él. Empecé un tercero y un cuarto, sólo que entonces alguien me miraba por encima del hombro y cuchicheaba con una voz aguda y clara como la nota de un violín: "Don Birnam", me susurraba, "no es lo bastante bueno. Así no. ¿Y si para entonarte te tomas un par de tragos, eh?”. Así que me tomé un par. iQué gran idea! Todo fue diferente. De repente, lo tenía todo claro. El trágico desarrollo de una gran novela, hermosamente proporcionada. Pero antes de dominarla y plasmarla sobre el papel, se me pasaba el efecto y todo se desvanecía como un espejismo. Entonces venía la desesperación, y un trago como contrapeso de la desesperación, y otro como contrapeso del contrapeso. Me sentaba frente a la máquina de escribir tratando de extraer una sola página que fuera medio decente, hasta que ese tipo volvía a aparecer.

$-\mathrm{H}$ : ¿Qué tipo, de quién estás hablando?

-D: El otro Don Birnam. Somos dos, ¿sabes? Don el borracho y Don el escritor. El borracho le decía al escritor: "Venga, idiota, saquemos algo en limpio de esa máquina, empeñémosla, llevémosla a la casa de empeño de la Tercera Avenida, seguro que nos dan diez dólares. Otra juerga, otra borrachera, otra trompa, otro sorbito... Palabras tan cómicas... Intenté alejarme de ese tipo muchas veces, pero... fue inútil. ¿Sabes? Una vez incluso me compré una pistola. Y balas. Iba a hacerlo cuando cumpliese treinta años. (Abre el cajón, saca dos balas, las sostiene en la palma de la mano.) Aquí están las balas; la pistola la cambié por una botella de whisky. El otro Don quiso que tomáramos una copa antes. Siempre quiere tomar una copa antes. El suicidio frustrado de un escritor frustrado (Wilder, 1945). ${ }^{1}$

Podría parecer, en un principio, que la frustración del escritor no es más que una excusa para explicar su alcoholismo, en la medida en que The Lost Weekend se centra en el descenso a los infiernos de un adicto que comienza engañando a su familia para que se marchen de viaje sin él y termina tratando de llevar a cabo

\footnotetext{
${ }^{1}$ La traducción de los diálogos, en todas las citas de la película, es del autor.
} 
el acto de suicidio al que había aludido en la escena citada - entre los dos extremos, el personaje ha tratado de robar el bolso de una desconocida, ha deambulado por la Tercera Avenida tratando de empeñar sin éxito su máquina de escribir, ha sido internado en el pabellón de alcoholicos de un hospital y ha sufrido un ataque de delirium tremens-. No obstante, la sombra de la escritura persiste en la película como algo más que una excusa: se trata, por el contrario, de un problema de identidad que persigue al protagonista hasta el último momento.

Permítaseme citar, sólo como anécdota, una secuencia de Historias de Filadelfia (The Philadelphia Story, George Cukor, 1940) en la que el elegante C. K. Dexter Haven (Cary Grant) declara que, según tiene entendido, "todos los escritores beben demasiado y pegan a sus esposas”. La imagen tópica del escritor alcoholizado se hizo muy popular por diversos factores, sobre todo por la famosa "Ley Seca" que prohibió la venta de alcohol entre 1920 y 1933, y que había sido efecto de las presiones de los grupos más conservadores de Estados Unidos -sobre todo, fundamentalistas cristianos-, lo que llevó a los intelectuales progresistas a hacer bandera del alcohol para protestar contra los códigos morales vigentes ${ }^{2}$. Los viajes a París de los más influyentes miembros de la "Generación Perdida" - pero también muchos otros, como los humoristas de la Tabla Redonda del Algonquin - reforzaron el estereotipo, pues la mayor parte de los escritores de la época se sintieron liberados de toda constricción al descubrir la vida nocturna parisiense, en la que el alcohol no sólo no estaba prohibido, sino que creaba fuertes lazos de camaradería entre todos los "ciudadanos del mundo" que habían llegado a la capital francesa desde los más dispares rincones de la Tierra. La asociación entre la literatura y el alcohol dio a éste un cierto prestigio, al menos hasta la aparición de Días sin huella, donde dicha asociación queda en entredicho, desde el momento en que el alcoholismo del escritor se convierte en efecto de su impotencia, consecuencia de un vacío interior que no le es posible llenar de otro modo:

- WICK (W): Puede que no seas escritor. ¿̇Por qué no haces otra cosa?

-DON (D): iClaro! Buscar un buen trabajo: funcionario público, agente inmobiliario... No tengo agallas, Helen. La mayoría de los hombres viven en una silenciosa desesperación. iYo no soporto la desesperación!

\footnotetext{
2 "By the end of the nineteenth century, however, the main population base for the temperance movement had shifted to fundamentalist and conservative Protestant denominations in the South and midwest. In the South, the movement had become identified with racism and with the maintenance of the Bourbon hegemony -the dominant Southern politics of the time, quite against the factual evidence, invoked and played upon an association of blacks with drunkenness [...]. In such a political climate, college students with vague liberal or radical political leanings were likely to come to regard visible drinking and drunkennes as an act of political dissent" (Room, 1984, p. 3).
} 
-Helen (H): iPero eres escritor! Tienes cualidades para ello: imaginación, comprensión...

-D: Vamos, seamos realistas. Tengo treinta y tres años, vivo de la caridad de mi hermano: pensión y alojamiento gratis, cincuenta centavos semanales para cigarrillos, una entrada para el teatro o un concierto, todo fruto de la grandeza de su corazón. Tiene un corazón grande y paciente...

-W: Sólo te mantengo provisionalmente.

-D: iCállate, Wick! Nunca he hecho nada, ahora no hago nada, y sé que nunca haré nada. iCero, cero, cero! (Wilder, 1945).

\section{La angustia de la escritura...}

Es preciso señalar, pues, que si bien Días sin huella sigue siendo una película sobre el alcoholismo, también plantea un problema asociado a la idea de la escritura, el conocido como "miedo a la página en blanco", y lo hace presentando al escritor como un ser desdoblado en la paradójica condición de escritor que no escribe. La publicidad que la Paramount dio a la película daba cuenta precisamente de este desdoblamiento al poner el acento en el oscuro sentido simbólico de la alucinación que, en pleno ataque de delirium tremens, experimenta Birnam, en la que un murciélago mata a un ratón que ha aparecido en un agujero de la pared (F1), tal como explica Ed Sikov:

El sanguinario murciélago y el pobre ratón se convirtieron en uno de los principales reclamos de la promoción que hizo la Paramount de Días sin huella. En 1944 y 1945, la teoría psicoanalítica freudiana estaba ganando muchos adeptos en Estados Unidos y la alucinación con roedores [sic] de Birnam caló profundamente en la imaginación popular. La gente de prensa de la Paramount fue más allá de su habitual cháchara halagadora al anunciar al público que la mente subconsciente del héroe expresa su complejo de inferioridad mediante el ratón, mientras que el murciélago es el escritor de éxito que Birnam se imagina que es cuando está borracho. "El ego más poderoso mata al más débil y ahí está el clímax dramático de la tragedia", explicaban los publicistas de la Paramount. El mismo Wilder dijo que Jackson le había explicado con detalle el simbolismo: "La alucinación de Birnam es una consecuencia de su esquizofrenia o personalidad escindida. El ratón representa al Birnam de cada día; el murciélago - o ratón con alas - al artista que sueña ser. El murciélago, claro, destruye al ratón. No, no lo explicamos en la película. Así damos a los espectadores la oportunidad para ejercitar la mente; además, no teníamos tiempo" (Sikov, 1998, p. 286).

La secuencia es, con todo, perturbadora, y el plano de Don Birnam chillando aterrorizado (F2) puede hacernos dudar de hasta qué punto puede considerarse positiva la imagen del escritor de éxito. El murciélago, animal tradicionalmente impuro y demoníaco, es percibido por Birnam como una amenaza, opuesta a la ternura que le había inspirado unos momentos antes el ratón de la pared. Podría pensarse, siguiendo los esquemas del psicoanálisis, que en realidad Birnam se sirve inconscientemente del alcohol para boicotear su carrera de escritor, 
atemorizado por las fuerzas oscuras que podrían darse a conocer a través de su escritura3.

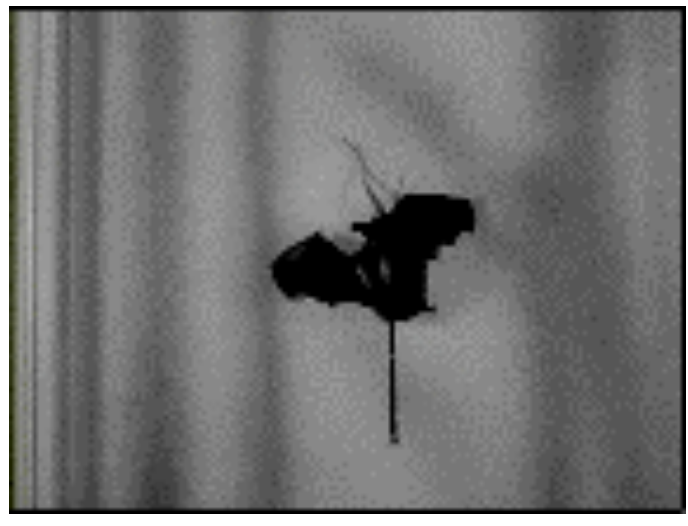

F1. Billy Wilder, The Lost Weekend, 1945

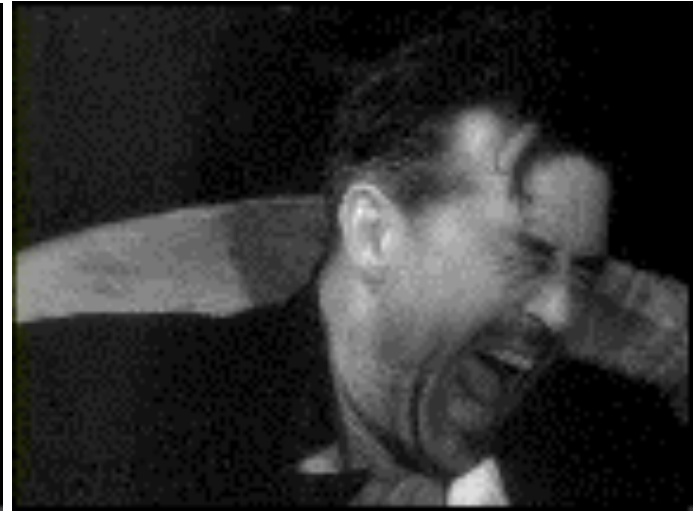

F2. Billy Wilder, The Lost Weekend, 1945

No obstante, esta interpretación puede resultar algo forzada, pues la imagen que proyecta Birnam no es la de un creador obsesionado por sus demonios internos 4 . Por el contrario, lo que le ocurre es que se halla paralizado por la presión social que implica la condición de escritor ante el público, presión muy real a la que quizás se pueda achacar los conocidos casos de escritores "secretos" como Emily Dickinson o Fernando Pessoa. La responsabilidad del escritor hacia su palabra, la exigencia de emplearla siempre como algo que trasciende el uso del lenguaje como simple instrumento comunicativo, puede llegar a producir una grave sensación de angustia vital. Sensación que se acentúa aún más al reconocer que la palabra, considerada por el escritor como algo que emana de lo más irreductible de su personalidad, queda, una vez emitida, fuera de su control, tal como explica Jacques Derrida:

Si la angustia de la escritura no es, no debe ser un pathos determinado, es porque aquella no es esencialmente una modificación o un afecto empíricos del escribir, sino la responsabilidad de esta angustia, de ese pasaje necesariamente estrecho de la palabra contra el que se lanzan y se obstaculizan entre sí las significaciones posibles. Se obstaculizan entre ellas, pero se apelan, también se provocan,

\footnotetext{
3 Precisamente, la teoría del "bloqueo del escritor" la daría a conocer el psicoanalista Edmund Bergler dos años después del estreno de Días sin huella, en 1947: "Bergler analyzed blocking in highly psychosexual terms, defining creative writing as an expression of unconscious defenses against oral-masochistic conflicts, and writer's block as the result of the breakdown of those defenses" (Rose, 1984, p. 13).

4 Esto no impide, por lo demás, que se pueda llegar a algunas conclusiones respecto a todo lo que proyecta la angustia en la existencia de Birnam: "Para los psicoanalistas la angustia es esencial en la experiencia clínica porque es la vía por la que un sujeto puede aprehender algo sobre su ser de goce, es decir, sobre su manera de gozar en la vida. Tanto de aquello que le hace sufrir, y de lo que él goza sin saberlo, como sobre su deseo inconsciente. Sin la angustia, no sabríamos nada sobre el deseo ni sobre el goce. La angustia es siempre la de un sujeto que habla y es a través de sus palabras como podremos cernir su causa" (Fuentes, 2017). Desde este punto de vista, podría decirse que el bloqueo de Birnam es fruto de un deseo masoquista que apunta más allá de su deseo de triunfar como escritor para llevarle hacia la autodestrucción por medio del alcohol.
} 
imprevisiblemente y como a pesar mío, en una especie de supercomposibilidad autónoma de las significaciones, potencia de equivocidad pura frente a la que la creatividad del Dios clásico parece todavía demasiado pobre. Hablar me da miedo porque, sin decir nunca bastante, digo también siempre demasiado. Y si la necesidad de hacerse soplo o palabra estrecha el sentido -y nuestra responsabilidad del sentido-, la escritura de nuevo estrecha y constriñe más la palabra (Derrida, 1967, pp. 17-18; cursiva en el texto).

La presión puede ser excesiva, y llevar a lo que se conoce como "síndrome de Bartleby" 5 , pero también puede dar lugar a otra reacción, la ya citada del miedo a la página en blanco, conocido igualmente como "bloqueo del escritor". La diferencia reside en que el creador afectado por este bloqueo no renuncia a su condición de escritor; por el contrario, lucha contra la presión de la escritura intentando dominarla de un modo u otro, tarea en la que puede salir victorioso - gracias a su inspiración o a su trabajo, o a una combinación de los dos-, pero también derrotado. Días sin huella no renuncia a presentar un fútil intento del personaje de comenzar la escritura de su novela continuamente postergada - titulada, significativamente, The Bottle-, en el que su trabajo no llegará más allá de la dedicatoria (F3). Birnam deja la máquina de escribir en seguida, y la cámara le sigue en una breve panorámica hasta que se sitúa frente a la botella vacía (F4), evidenciando que "Don el escritor" ha sido sustituido por "Don el borracho", que abandona la tarea iniciada para dedicarse a buscar la botella escondida el día anterior, cuyo paradero es incapaz de recordar.
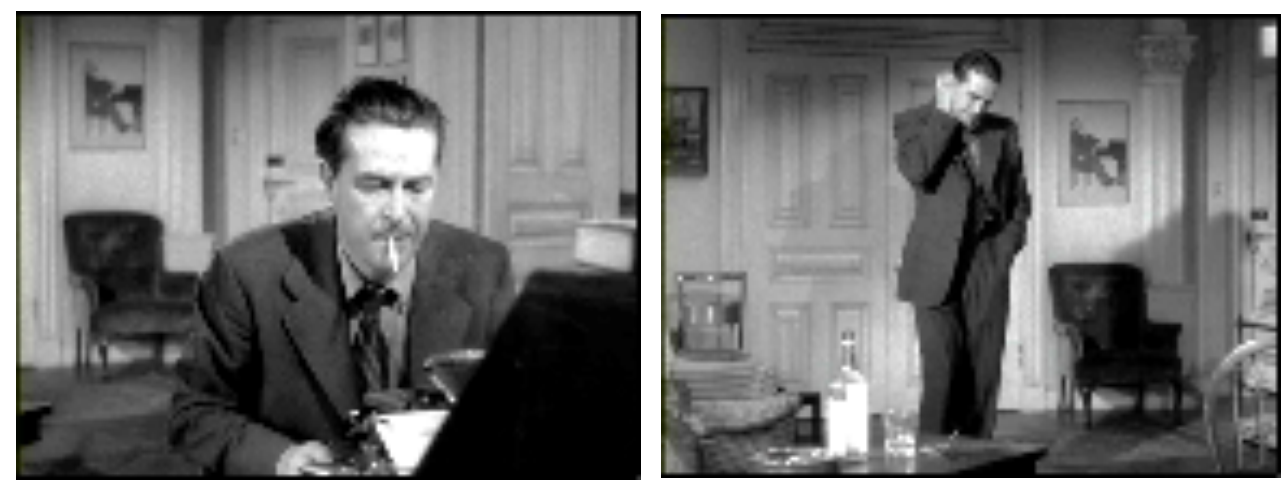

F3. Billy Wilder, The Lost Weekend, 1945 F4. Billy Wilder, The Lost Weekend, 1945

\section{3. ...vs. la vocación del escritor}

Parecería, pues, que el escritor sólo existe cuando el borracho desaparece, y viceversa. En realidad, no es así: la imagen tópica de la escritura es la de una

\footnotetext{
5 Enrique Vila-Matas lo define, en Bartleby y compañía, como "la pulsión negativa o la atracción por la nada que hace que ciertos creadores, aun teniendo una conciencia literaria muy exigente (o quizás precisamente por eso), no lleguen a escribir nunca; o bien escriban uno o dos libros y luego renuncien a la escritura; o bien, tras poner en marcha sin problemas una obra en progreso, queden, un día, literalmente paralizados para siempre” (Vila-Matas, 2000, p. 12).
} 
vocación a la que es imposible resistirse. Según esta noción, el escritor oscila -y aquí es donde emerge la angustia - entre el placer de su actividad y el sufrimiento que le produce todo lo que le aparta de ella, entre la plenitud de su labor y la vacuidad de su existencia cuando no puede llevarla a cabo. La paradoja del escritor residiría en que sólo puede serlo con total intensidad en el presente de su trabajo, mientras que cualquier proyección hacia el pasado - "yo escribía" - o hacia el futuro -"yo escribiré" - resultará engañosa, pues ni en la memoria ni en la imaginación se puede experimentar la felicidad que confiere lo absoluto del presente, donde el sujeto se experimenta en su totalidad. En ese sentido, no escribir implica el dolor de renunciar al yo, no poder experimentarlo en el apogeo de ese presente activo que es la operación de escribir (véase Heinich, 2000, pp. 104 y ss.).

Un escritor que, parafraseando a Karl Kraus, tuviera "carácter suficiente para no escribir” (Kraus, 1924, p. 122) podría renunciar a la escritura, siguiendo así los pasos de los afectados por el "síndrome de Bartleby". Pero ya hemos visto que éste no es el caso de Birnam, que se debate entre el deseo y la impotencia de ser y no ser a la vez escritor. Y si bien es el desaliento el que le empuja a la bebida, llegando en ocasiones a renegar de sí mismo, lo cierto es que su vocación no le abandona en ningún momento. La imagen arquetípica del artista moderno le exige que sea escritor a tiempo completo $^{6}$, y así parecen reconocerlo Wilder y Brackett en lo que se ofrece de forma sencilla al espectador como una argucia del guión para plantear una elipsis7, pero termina siendo un reconocimiento de la condición vocacional de la escritura.

En su primera visita al bar, después de haber sucumbido por fin a la tentación, Birnam pide al barman que no elimine el círculo líquido que ha quedado tras retirar el vaso: "No lo limpies, Nat. Déjame conservar mi círculo del vicio. ¿Sabes? El círculo es la figura geométrica perfecta. Sin fin ni principio" (F5). Tras una secuencia en la que las conversaciones de Birnam con Nat (Howard da Silva) y con una joven prostituta, Gloria (Doris Dowling) se formulan mediante la técnica

\footnotetext{
${ }^{6}$ Recuérdese, por ejemplo, el brillante relato de Henry James "La vida privada" (1892), en el que también se especula en torno al desdoblamiento del escritor, dividido en dos instancias: la figura pública que brilla en las cenas de sociedad y el artista genial que trabaja en la soledad de su cuarto. El fantástico descubrimiento del narrador, al comprobar que el dramaturgo Clare Vawdrey está en los dos lugares al mismo tiempo, puede leerse como una declaración de principios del propio James - quien también llevaba una vida social muy activa-, en el sentido de que el escritor no deja de serlo en ningún momento, aunque parezca que los momentos en que no escribe le alejan de su condición.

7 Según declaraba Wilder, "utilicé este fundido encadenado, del que realmente estoy un poco orgulloso, para ganar tiempo, y para no tener que contar de un modo aburrido: primero se toma una copa, después no puede detenerse y se toma otra y otra. La breve sucesión de círculos da a entender claramente al público, de golpe, la derrota de un bebedor reincidente" (Wilder \& Karasek, 1992, p. 236).
} 
del plano/contraplano, una elipsis salta un par de horas para mostrar los círculos que se han ido acumulando sobre la barra.

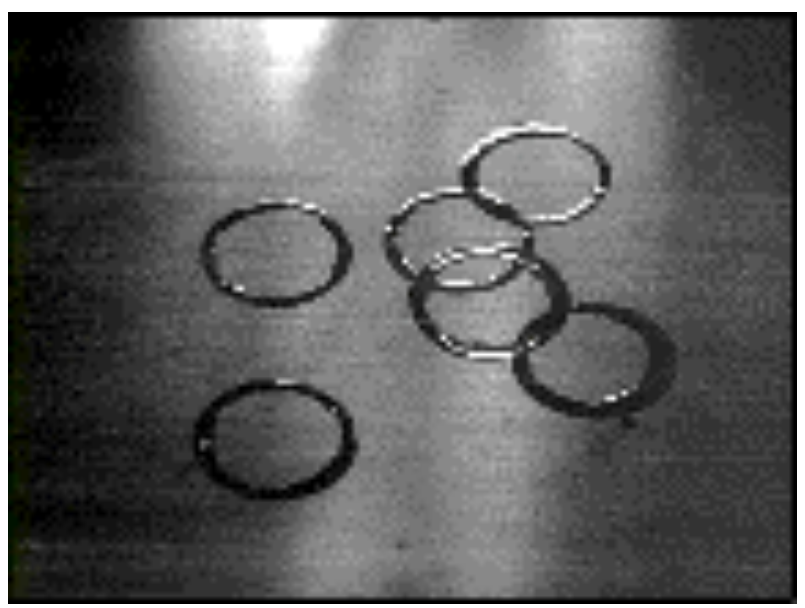

F5. Billy Wilder, The Lost Weekend, 1945

Es evidente que el círculo vicioso al que alude el propio Birnam es el de su alcoholismo, que la película va a mostrar en toda su crudeza, comenzando por la suma de círculos que se va dibujando en la barra conforme pasa el tiempo. Sin embargo, resulta difícil aceptar que sea en estas circunstancias que el alcohólico desplace al escritor cuando, movido por la euforia que provocan en él las primeras copas, comienza a fantasear sobre los efectos que la bebida produce en su personalidad:

-Don: Me encoge el hígado, ¿̇verdad, Nat? Me destroza los riñones, sí. ¿̇ero qué le hace a mi cabeza? Lanza los sacos de arena por la borda para que el globo se alce... iY no soy un tipo corriente, me siento preparado, muy preparado! Cruzo las cataratas del Niágara como un funámbulo, soy uno de los grandes genios. Soy Miguel Ángel modelando la barba de Moisés. Soy Van Gogh pintando la luz del sol. Soy Horowitz tocando el Concierto Emperador. Soy John Barrymore antes de que el cine lo ahogara. Soy Jesse James y sus dos hermanos, ilos tres! iSoy William Shakespeare! Y aquello de ahí fuera ya no es la Tercera Avenida, ies el Nilo, Nat! El Nilo, y por él se desliza la barcaza de Cleopatra. Acércate... Las velas de púrpura, y tan perfumadas que los vientos languidecían de amor por ellas... Los remos, que eran de plata, acordaban sus golpes al son de flautas... (Wilder, 1945).

Como puede verse, más que extinguir su vocación literaria, lo que hace el alcohol es excitarla, aunque sin permitirle encontrar su propia voz, ya que no logra ir más allá de citar a Shakespeare (Antonio y Cleopatra, II, 2). La verdadera escritura de Birnam se encuentra, por tanto, en los círculos sin limpiar de la barra del bar, que se leen como un registro - una escritura, en definitiva- de su alcoholismo. En este sentido, los círculos no sólo funcionan semióticamente como índices que consignan el paso del tiempo y el número de copas consumidas por Birnam; también aluden a su impotencia para trazar otros signos que no sean estos de carácter circular. 


\section{Aprender a leer los signos}

El problema de Birnam, en realidad, es su incapacidad para reconocer el valor de los signos: a diferencia del espectador, que reconoce el incremento de los círculos de la barra como índice de una elipsis, Birnam ignora esta semiosis - un plano medio del personaje permite ver la barra del bar, con varios bebedores concentrados en sus copas - y prosigue con su perorata, hasta que es el barman quien le avisa de que se le hace tarde - la imagen corta a la salida del bar, en un travelling lateral que sigue a un Birnam apresurado y tambaleante-. Ha de entenderse, por tanto, que el efecto que el alcohol produce en Birnam no es el de anularle como escritor - la vocación permanece activa en todo momento-, sino el de impedirle alcanzar el verdadero sentido de la escritura: un lastre que debe soltar si quiere convertirse en un escritor genuino, más allá de las citas shakespearianas. Así pues, sin dejar de ser el relato de la degradación de un alcohólico, la película mostrará también el necesario aprendizaje de Birnam para alcanzar el objetivo deseado de una escritura auténtica.

La lección consistirá en aprender a leer los signos de forma correcta. Una de las secuencias más reconocidas de la película es aquella en la que Birnam descubre la botella que había ocultado el día anterior en la lámpara del techo. Después de haber revuelto toda la casa en una ansiosa búsqueda, después de haberse visto sorprendido tras robar un bolso en un club nocturno, Birnam regresa a casa y se tumba en su cama. Su posición le permite descubrir la botella, gracias a la sombra que proyecta hacia el techo, evidenciando que, a diferencia de lo que había ocurrido con los círculos de la barra del bar, Birnam ha sabido reconocer la condición de signo -índice, como en el caso anterior- de la sombra del techo. El cuello de la botella que asoma por encima de la lámpara permite además, por si quedaba alguna duda, mostrar el significado del signo en su relación con el significante (F6).

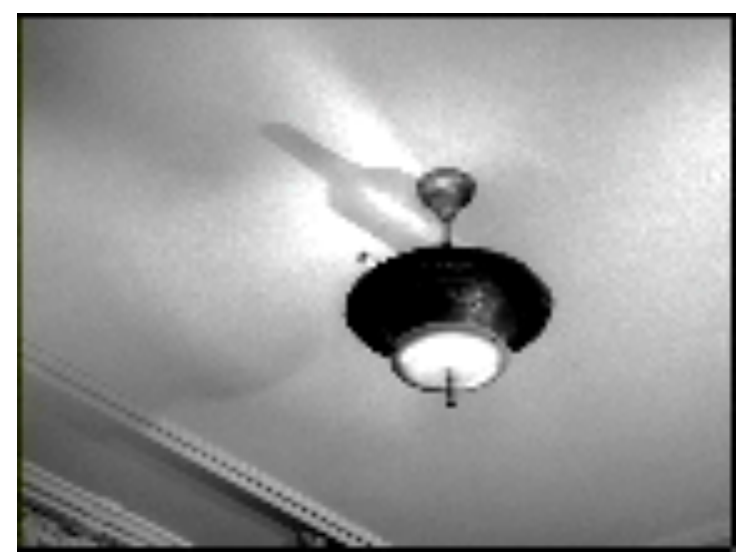

F6. Billy Wilder, The Lost Weekend, 1945 
Birnam volverá a encontrarse con los signos durante su estancia en el pabellón de alcohólicos del hospital, durante el ataque de delirium tremens de uno de los internos, del que se ha dicho que en sus desvaríos se ve rodeado de escarabajos. Los espasmódicos movimientos del paciente - que se muestran a través de un plano entero de carácter subjetivo, siguiendo la mirada de Birnam - pueden ser entendidos, al igual que los signos analizados hasta ahora, como índices de su estado alucinatorio, síntomas de su enfermedad. No obstante, el detalle de los escarabajos nos permite avanzar al terreno de los símbolos, ya que la relación entre significante y significado es en este caso arbitraria, lo que Birnam podrá experimentar en carne propia cuando sufra su propio ataque de delirium tremens, esta vez con un ratón y un murciélago fruto de la imaginación de Birnam, mostrado en esta ocasión - al igual que ocurría con el paciente de los escarabajos, el ratón y el murciélago aparecen en planos subjetivos contrapicados, pero la mirada de Birnam se muestra a través de primerísimos planos frontales-. La diferencia entre las dos alucinaciones reside en que en la primera no se ven los escarabajos que atormentan al paciente, mientras que en la segunda sí se perciben los animales: efecto en los dos casos de la planificación subjetiva que permite al espectador ver lo mismo que está viendo Birnam. Evidentemente, el paso del "no ver" al "ver" es lo que lleva al personaje - al igual que a los espectadores - a la conclusión de que ha tocado fondo, razón por la que retomará su plan de suicidio.

Como ya se señaló antes, la alucinación de Birnam es susceptible de ser interpretada en un sentido plenamente simbólico. Es posible concluir, por tanto, que el aprendizaje del escritor ha recorrido un trayecto que va de la indiferencia hacia los signos - los círculos de la barra del bar cuyo significado es incapaz de percibir, distraído por su cháchara pseudoliteraria, que sólo ve en ellos la "figura geométrica perfecta” - a la comprensión de todo lo que éstos pueden llevar consigo. Su vocación de escritor puede manifestarse ahora en toda su plenitud, dejando atrás la escisión de su personalidad que le llevaba a beber. La conclusión de la película parece apuntar en esta dirección: Birnam se decide a dejar el alcohol para poner por escrito su peripecia durante el infernal fin de semana que acaba de pasar. La acción de tirar su cigarrillo dentro del vaso de whisky - una secuencia plano/contraplano entre Don y Helen, subrayada por la dramática música de Miklós Rózsa, muestra primero la aproximación de Don al vaso medio lleno de licor, la angustia de Helen ante la duda de si Don va a volver a la bebida, la acción de tirar el cigarrillo en la bebida y la sonrisa aliviada de Helen - parece bastante concluyente, sobre todo porque va acompañada de la decisión de retomar, ahora ya definitivamente, la escritura de su novela sobre el alcoholismo. 
Es posible, pues, que su fin de semana perdido le haya inspirado valor para embarcarse en una tarea que le permita resistir la tentación.

Este final no debe extrañarnos, ya que el axioma del cine clásico sobre la escritura insiste una y otra vez en la necesidad del escritor de centrarse en la performatividad del discurso ${ }^{8}$. En este caso, Birnam encuentra las fuerzas necesarias para superar su miedo a la página en blanco transfigurando su escritura en una misión moral, la de hacer que el público tome conciencia del problema social del alcoholismo. La película termina con una panorámica que va de la botella que tenía oculta Birnam al comienzo del relato - lo individual- al plano general de la ciudad de Nueva York - lo colectivo-, mientras se oye la voz en off del futuro autor de la novela en la que se basará la película: "Me pregunto cuántas personas hay como yo. Pobres tipos envilecidos, ardiendo de sed. Personajes cómicos para el resto del mundo cuando se tambalean a ciegas, buscando otro trago, otra borrachera, otra juerga”.

\section{Conclusiones}

Como puede verse, era necesario que Birnam aprendiese a reconocer el valor de los signos, pues su escritura sólo encontrará sentido en el momento en que se postule a sí mismo como portavoz de los alcohólicos para denunciar su dramática condición. Para ello no basta únicamente con transcribir la propia experiencia - el primer proyecto de Birnam se titulaba The Bottle-, sino que es preciso que su relato - ya diferente de la propuesta inicial, ahora con el título de The Lost Weekend- adquiera simbólicamente la condición de experiencia colectiva, valiosa para toda la comunidad. Símbolo que incluye, debe incluir, la redención final del alcohólico: "No pudiste escribir el comienzo porque no conocías el final”, dice Helen aludiendo a los fracasos previos del escritor, pero augurando a la vez el triunfo final de "Don el escritor" sobre "Don el borracho". La panorámica final - de la botella colgada en la ventana (F7) al paisaje neoyorkino (F8) - realiza el recorrido contrario que hacía la panorámica inicial de la película - del paisaje a la botella-, repitiendo el gesto, pero en sentido inverso, para clausurar así el relato, ofreciendo una metonimia del propio discurso cinematográfico que se podría leer a modo de frase hermenéutica bathesiana. No obstante, esta escena final se ofrece al espectador como un flashback que le lleva al comienzo de la narración, dando a ésta una estructura circular de myse en abime: la novela que proyecta Birnam -"voy a escribir todo este fin de semana" (Wilder, 1945) - remite, pues, a la novela The Lost Weekend de

8 Para esta cuestión, véanse mis artículos sobre oralidad y escritura en la película de William Dieterle Esmeralda la zíngara (Pueo, 2006) y en la de Alfred Hitchcock Enviado especial (Pueo, 2017). 
Charles R. Jackson, que llevada al cine da lugar a la película del mismo título, en la que de nuevo se presenta el caso de Birnam, y así sucesivamente. No se necesitan más pistas para entender que la película se presenta como el fruto de la escritura, ya liberada del alcohol, de Don Birnam. Las declaraciones realizadas por Billy Wilder respecto a la redención del alcohólico no fueron, sin embargo, nada concluyentes: "para mí, la película termina con un punto de esperanza: Birnam empieza a escribir de nuevo. Pero en realidad, el final es incierto; en cualquier momento puede darse la habitual recaída" (Wilder \& Karasek, 1992, p. 234). A pesar de ello, la existencia de un relato cinematográfico con el título de The Lost Weekend permite concluir que Birnam ha dejado el alcohol, al menos durante el tiempo necesario para liberarse del miedo a la página en blanco y poder escribir su novela.

\section{Referencias bibliográficas}

Bordwell, D. (1985). La narración en el cine de ficción. Barcelona, Paidós, 1996.

Bordwell, D. (1989). El significado del filme. Barcelona, Paidós, 1995.

Bordwell, D., Staiger, J. \& Thompson, K. (1985). El cine clásico de Hollywood. Barcelona, Paidós, 1997.

Derrida, J. (1967). La escritura y la diferencia. Barcelona: Anthropos, 1989.

Fuentes, A. (2017). ¿Por qué la angustia no tiene sentido, pero sí causa?, La Vanguardia,

https://www.lavanguardia.com/vida/20170126/413686201444/angustia-eldivan-afliccion-psciologia-psicoanalisis.html (última consulta, 17-122018).

Heinich, N. (2000). Être écrivain. París: La Découverte.

Jackson, C. R. (1944). Días sin huella. Barcelona: Argos Vergara, 1981.

James, H. (1892): La vida privada, en Los papeles de Aspern. Barcelona: Seix Barral, 1985.

Kraus, K. (1924). Dichos y contradichos. Barcelona: Minúscula, 2003.

Metz, C. (1968-73). Ensayos sobre la significación en el cine. Barcelona, Paidós, 2002.

Metz, C. (1977). El significante imaginario: psicoanálisis y cine. Barcelona, Paidós, 2001.

Pueo, J. C. (2006). “'ANÁГKH”, Escritura e imagen, 2, 2006, pp. 23-39.

Pueo, J. C. (2017). Vientos de guerra: oralidad y escritura en Enviado especial (Alfred Hitchcock, 1941), Tropelías, núm. extraordinario 2, Homenaje a José Antonio Pérez Bowie, pp. 462-471.

Room, R. (1984). A "Reverence for Strong Drink": the Lost Generation and the Elevation of Alcohol in American Culture, Journal of Studies on Alcohol, 45/6, pp. 540-546. 
https://www.robinroom.net/reverenc.htm (última consulta, 6-4-2018).

Rose, M. (1984). Writer's Block: The Cognitive Dimension. CarbondaleEdwarsville: Southern Illinois University Press.

Sikov, E. (1998). Billy Wilder: vida y época de un cineasta. Barcelona: Tusquets, 2000.

Vila-Matas, E. (2000). Bartleby y compañía. Barcelona: Anagrama, $5 \cdot^{\mathrm{a}}$ ed., 2002.

Wilder, B. \& Karasek, H. (1992). Nadie es perfecto. Barcelona: Grijalbo, $4 .^{\text {a }}$ ed., 1994.

Žižek, S. (1991). Mirando al sesgo. Barcelona, Paidós, 2000.

\section{Filmografía}

Wilder, Billy (Director) (1945). The Lost Weekend. Estados Unidos: Paramount Pictures. 